\title{
CIRCULAR DICHROISM STUDY OF THE LIGHT-HARVESTING PCP COMPLEXES WITHIN THE VIBRONIC MULTISTATE DIMER THEORY*
}

\author{
M. Pilch and M. PaWlikowski \\ Department of Theoretical Chemistry, Jagiellonian University \\ Karasia 3, 30-060 Kraków, Poland \\ (Received August 12, 1990)
}

\begin{abstract}
Chiral properties of peridinin-chlorophyll-protein (PCP) light-harvesting complexes are studied in terms of vibronic dimer theory previously applied to study certain structural aspects of $\alpha$-crustacyanin pigments. On the base of CD spectra it is shown that the peridinin dimer acts as a chiral group in PCP complexes and its geometrical structure is such that the peridinin monomers cannot be coplanar. Certain observations concerning the energy transfer process in PCP complexes in vivo are also made.
\end{abstract}

PACS numbers: 33.10.Lb, 33.55.Ad

\section{Introduction}

The circular birefringence phenomenon has a long history going back to the first Arago's[1] and Biot's[2] optical experiments. However, only during the last four decades the optical rotatory dispersion (ORD) and circular dichroism (CD) have been widely used to study a huge number of molecules which are capable to interact non-equivalently with the left and right-handed circularly polarized light. Such molecules are said to be optically active and must satisfy well-known stereochemical rule which states: only molecules not superimposable on their mirror images can show an optical activity. Most of biologically important molecules obey this general rule making ORD and CD spetroscopies extremely useful for studying enormous number of biological systems in vitro as well as in vivo [3, 4].

The photosynthetic light-harvesting peridinin-chlorophyl-proteins (PCP) complexes serve here as an obvious example. In these complexes the solar energy

*This work was supported by the project CPBP 01.12 
absorbed by carotenoid (peridinin) is transferred to the chlorophyll-a and the efficiency of this process depends on the mutual orientation of peridinin molecules. The circular dichroism (CD) analysis has shown that the efficient energy transfer in PCP complexes can occur when the peridin molecules are arranged as dimers (see Fig. 1) and monomers are oriented in such a way that their axes are almost perpendicular. The similar dimeric structures of astaxantin molecules were found in $\alpha$-crustacyanin responsible for the bluish colour of the lobster shell, (Homarus gammarus) $[7,8]$. Although the PCP complex obtained from marine dinoflagellate algae and $\alpha$-crustacyanin pigment extracted from lobster shell differ essentially in the chemical sense, the dimeric structure of their optically active groups is similar and somehow unique $[5,7]$. Some structural aspects of astaxantin dimers accommodated in $\alpha$-crustacyanin have been already studied theoretically in connection with chiral properties of these dimers [8]. It seems that the role played in plants by the light-harvesting systems makes the similar studies for PCP complexes desirable.

The aim of this paper is to discuss in some details CD and absorption spectra of PCP complexes extracted from Amphidinium carterae and Amphidinium rhyncocephaleum dinoflagellate algae. To this end we use the vibronic dimer theory formulated and applied earlier $[8,12]$. In particular we wish to show that the principal chiral properties of PCP complexes observed in the range of $15000-25000 \mathrm{~cm}^{-1}$ can be understood when the peridinin dimer acting in PCP as prosthetic group has the geometrical structure as shown in Fig. 1.

\section{Method of calculation}

Quantum mechanical theory of circular dichroism phenomenon[9] teaches us that absorption and circular dichroism associated with a vibronic transition $\Psi_{0} \longrightarrow$ $\Psi_{\nu, \lambda}$ can be characterized by the absorption coefficient $D(0 \longrightarrow \nu, \lambda)$ and the rotatory strength $R(0 \longrightarrow \nu, \lambda)$ calculated from

$$
\begin{aligned}
& D(0 \longrightarrow \nu, \lambda)=\left(\left\langle\Psi_{0}|\widehat{D}| \Psi_{\nu, \lambda}\right\rangle\right)\left(\left\langle\Psi_{\nu, \lambda}|\widehat{D}| \Psi_{0}\right\rangle\right) \\
& R(0 \longrightarrow \nu, \lambda)=\operatorname{Im}\left(\left\langle\Psi_{0}|\widehat{D}| \Psi_{\nu, \lambda}\right\rangle\right)\left(\left\langle\Psi_{\nu, \lambda}|\widehat{M}| \Psi_{0}\right\rangle\right)
\end{aligned}
$$

where $\widehat{D}$ and $\widehat{M}$ are the electric and magnetic dipole operator, respectively. The brackets \langle\rangle and ( ) denote integration over electronic and vibrational coordinates, respectively, and indices $\nu$ and $\lambda$ specify the vibronic levels of a molecule. In a dimer the vibronic states $\Psi_{\nu, \lambda}$ with $\lambda=+$ and - are symmetrical and antisymmetrical with respect to permutational operator $\widehat{G}$.

In this paper we are interested in the transitions from the totally symmetric ground state which is well separated on the energy scale from the excited electronic states of the dimer. So, we safely assume that $\Psi_{0}$ can be adequately represented in the Born-Oppenheimer approximation. It does not apply to the excited states because $\Psi_{\nu, \pm}$ must account for vibronic coupling [10] as well as the couplings of the excited electronic states introduced by the monomer-monomer interaction operator $\widehat{V}_{\text {int }}[12]$. Thus, for a given vibronic transition, the absorption and rotatory 
strength expressions(1) will contain not one [11] but many terms of different electronic origin. For a dimer with achiral monomers we can write $[12,13]$

$$
\begin{aligned}
& D(0 \longrightarrow \nu, \pm)=\sum \sum_{i \leq j} D(i, j, \pm)\left(\Phi_{0} \mid \Phi_{\nu}^{i \pm}\right)\left(\Phi_{\nu}^{j \pm} \mid \Phi_{0}\right), \\
& R(0 \longrightarrow \nu, \pm)=\sum \sum_{i \leq j} R(i, j, \pm)\left(\Phi_{0} \mid \Phi_{\nu}^{i \pm}\right)\left(\Phi_{\nu}^{j \pm} \mid \Phi_{0}\right),
\end{aligned}
$$

where the sums run over an arbitrary number of monomer states contributing to interstate couplings. $D(i, j, \pm)$ and $R(i, j, \pm)$ depend on the symmetry of the monomer electronic states as well as on the geometry of the dimer. For the purpose of the next section we assume that only two electronic states, say, $|m\rangle$ and $|n\rangle$ in the monomer are involved in monomer-monomer interaction and that the transition dipole moments to these states are mutually parallel. Then applying expressions (14) from Ref. [13] to the dimer geometry in Fig. 1, we get

$$
\begin{array}{r}
R(i, j, \pm)=\frac{1}{\sqrt{2}}\left(1-\frac{1}{2} \delta_{i j}\right)|R| D_{i} D_{j} \sin \beta \\
D(i, j, \pm)=\left(2-\delta_{i j}\right) D_{i} D_{j}(1 \pm \cos \beta)
\end{array}
$$

where $D_{i}$ for $i=m$ and $n$ are the "lengths" of transition dipole moment for $|g\rangle \longrightarrow|i\rangle$ electronic excitation in monomer.

With the electronic origin factors (3) the absorption and rotatory strengths could be calculated if the vibrational overlap integrals $\left(\Phi_{0} \mid \Phi_{\nu}^{i \pm}\right)$ and $\left(\Phi_{\nu}^{j \pm} \mid \Phi_{0}\right)$ were known. These integrals can be simply found since the vibrational functions $\Phi_{\nu}^{m \pm}$ and $\Phi_{\nu}^{n \pm}$ satisfy the vibronic equation [12]

$$
\left[\begin{array}{cc}
\widehat{h}_{m} \pm V_{m} \widehat{G}, & \pm V_{m n} \widehat{G} \\
\pm V_{m n} \widehat{G}, & \hat{h}_{n} \pm V_{n} \widehat{G}
\end{array}\right] \cdot\left[\begin{array}{c}
\Phi_{\nu}^{m \pm} \\
\Phi_{\nu}^{n \pm}
\end{array}\right]=E_{\nu}^{ \pm}\left[\begin{array}{c}
\Phi_{\nu}^{m \pm} \\
\Phi_{\nu}^{n \pm}
\end{array}\right]
$$

which is readily numerically solvable for given values of the resonance interacting energics (Davydov splittings) $V_{m}$ and $V_{n}$ as well as the interstate coupling parameter $V_{m n}[12,13]$. Dealing with equation (4) we have assumed that the monomer has only single totally symmetric vibration with the normal coordinate $Q$ and the excited states $|m\rangle$ and $|n\rangle$ undergo the Franck-Condon displacements $B_{m}$ and $B_{n}$ along $Q$-coordinates upon electronic excitations. It amounts to the assumption that the Hamiltonians $\widehat{h}_{m}$ and $\widehat{h}_{n}$ in Eq. (4) can be written as

$$
\widehat{h}_{i}=E_{i}^{0}+\frac{1}{2} \sum_{\lambda=+,-}\left(\widehat{P}_{i}^{2}+\omega^{2} Q_{i}^{2}+\sqrt{2} B_{i} Q_{\lambda}\right),
$$

where symbols have their usual meaning. Here and hereafter the unit $\hbar=1$ is used.

Within the formalism sketched above, we are ready to discuss CD and absorption spectra of PCP complexes. Comparing the experimental spectra to the theoretical ones we will simulate these former as 


$$
\begin{aligned}
& R(\Omega)=\sum_{\nu} \sum_{\lambda=+,-} R(0 \longrightarrow \nu, \lambda) f_{\nu, \lambda}(\Omega), \\
& I(\Omega)=\sum_{\nu} \sum_{\lambda=+,-} D(0 \longrightarrow \nu, \lambda) f_{\nu, \lambda}(\Omega),
\end{aligned}
$$

where $f_{v, \lambda}(\Omega)$ is the normalizad to unity band-shape function assumed to be Gaussian

$$
f_{\nu, \pm}(\Omega)=\frac{1}{\Gamma_{\nu}^{ \pm} \sqrt{\pi}} \exp \left[-\left(\left(E_{\nu}^{ \pm}-\Omega\right) / \Gamma_{\nu}^{ \pm}\right)^{2}\right]
$$

$\Omega$ is the energy of absorbed light and $\Gamma_{\nu}^{\lambda}$ is the line-width of $\nu, \lambda$-th level.

\section{Result and discussion}

Peridinin-chlorophyl-protein (PCP) complexes acting in vivo as photosynthetic antenna pigments, contain one chlorophyll-a and an average number of four peridinin molecules per protein. Following earlier suggestions [5], we could assume that four peridinin molecules form pairs of weakly interacting dimers each having geometrical structure shown in Fig. 1. The low-temperature absorption spectrum

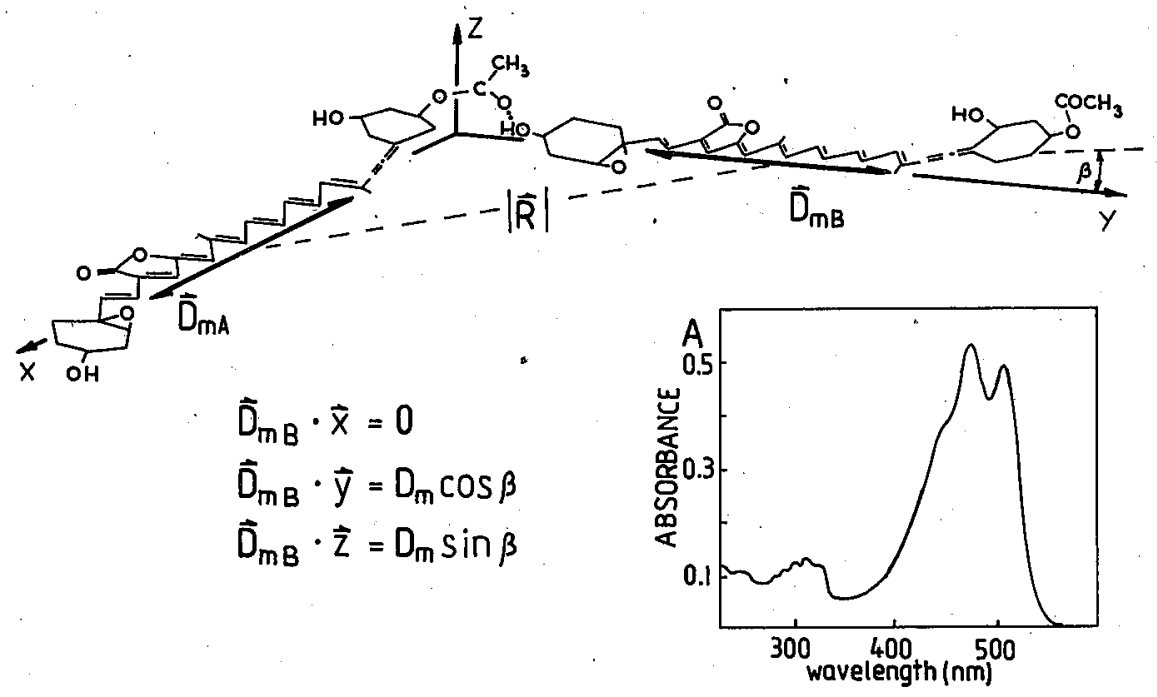

Fig. 1. A probable structure of peridinin dimer in PCP complex with $|\mathbb{R}|=12 \AA$, $\beta=20^{\circ}$ and the absorption spectrum of the isolated peridinin (inset).

of peridinin molecule is given in the inset of Fig. 1. Because in this absorption region, peridinin alone shows no $C D$ it is reasonable to assume that the chiral structure of peridinin dimer is responsible for the observed circular dichroism of 
PCP complexes. For PCP from A. carterae, CD and absorption spectra are shown as dot-dashed lines in Fig. 2. For the comparision the CD and absorption spectra of chlorophyll-a are also given. As can be seen from Fig. 2 the large CD signal observed for PCP complex could not be attributed to the chirality of the chlorophyll-a in the spectral region of interest.

Following these preliminary remarks we can now apply the theory outlined in previous section to reconstruct CD and absorption spectra given in Fig. 2.
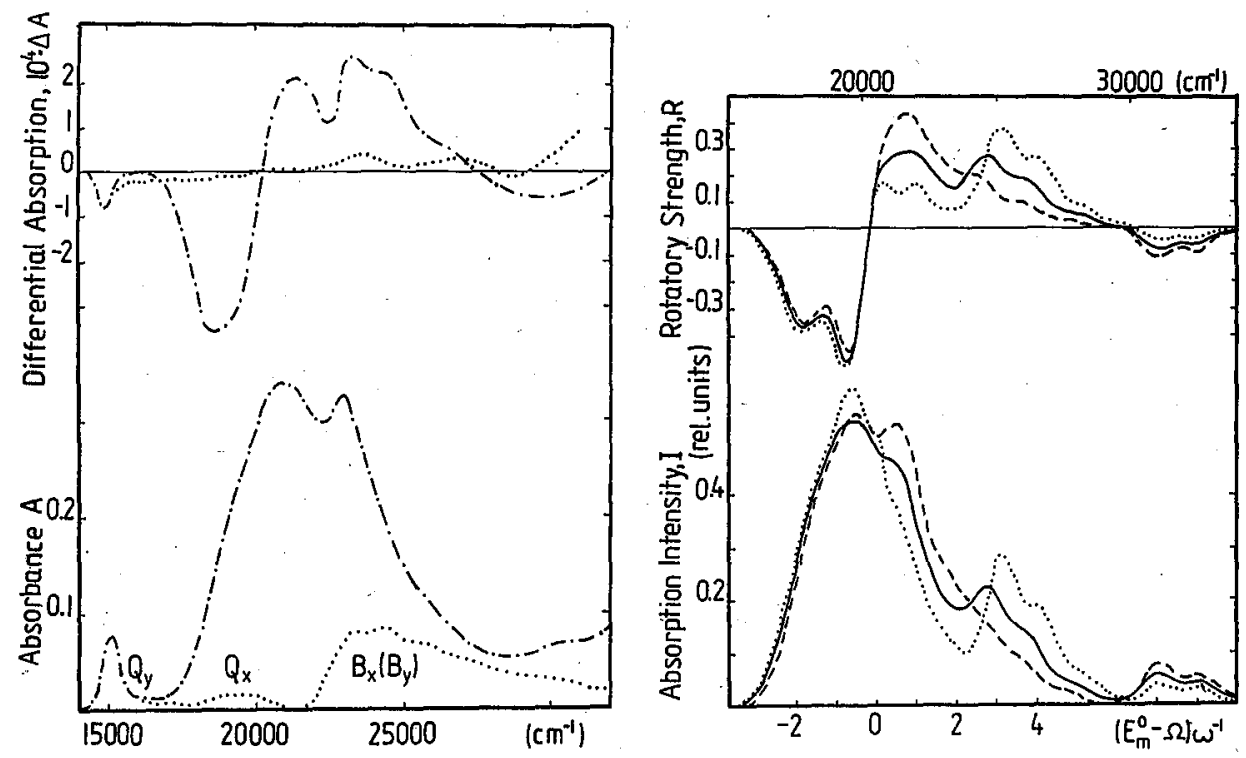

Fig. 2. CD and absorption spectra of chloroplyll a (...) and PCP complex from $A$. carlerae (-..-) [5].

Fig. 3. The theoretical $\mathrm{CD}$ and absorption spectra calculated for $V_{m n}=\omega(.$.$) ,$ $V_{m n}=0.5 \omega(-) \cdot V_{m n}=0(---)$ and the dimer geometry shown in Fig. 1. The remaining parameters are $\omega=1500 \mathrm{~cm}^{-1}, E_{n}-E_{m}=5.5 \omega, D_{n} / D_{m}=0.4, B_{m}=1.5 \omega^{3 / 2}$, $B_{n}=1.65 \omega^{3 / 2}, V_{m}=0.7 \omega$ and $V_{n}=-2 \omega . \Gamma_{0}^{-}=0.9 \omega$ and $\Gamma_{i}^{ \pm}=0.55 \omega$ for all other lines.

The results are presented in Fig. 3. Except for the electronic integrals $V_{m}$, $V_{n}$ and $V_{m n}$, most parameters required in calculations could be evaluated from the absorption spectra of peridinin shown in Fig. 1 (inset). This spectrum reveals two electronic bands which can be assigned to ${ }^{1} A_{1} \rightarrow{ }^{1} B(m)$ (low-energy band) and ${ }^{1} A_{1} \rightarrow^{1} B(n)$ (high-energy band) excitations in the $\pi-\pi^{*}$ electronic system of carotenoid chain. From the well-resolved Franck-Condon progression of totally symetric vibration with $\omega=1500 \mathrm{~cm}^{-1}$, the displacement parameters $B_{m}=1.5$ $\omega^{3 / 2}$ and $B_{n}=1.65 \omega^{3 / 2}$ could be extracted. Finally, an analysis of the zeroth and first spectral moments of absorption bands yields of $D_{m} / D_{n}=0.4$ and $E_{n}^{0}-E_{m}^{0}=$ $5.5 \omega$. 
With these values of parameters and for the electronic matrix elements $V_{m}=$ $0.7 \omega$ and $V_{n}=-2 \omega$, the absorption and CD spectra are given in Fig. 3 for some values of interstate coupling matrix element $V_{m n}$. Due to an apparent absence of crystallographic data the structural parameters $\beta$ and $|\boldsymbol{R}|$ could not be precisely determined for PCP complexes. Nevertheless, we think that the values $\beta=20^{\circ}$ and $|R|=12 \AA$ used in the actual calculations are reasonable being consistent with those estimated from CD analysis for $\alpha$-crustacyanin [8]. It should be also noted that the angle $\beta$ has to differ from zero to make peridinin dimer optically active.

Comparing dot-dashed spectra in Fig. 2 with those in Fig. 3 one can see that somehow simplified dimer theory reproduces quite correctly the overall intensity distribution of the experimental spectra. In particular, the intensity ratio of two positive Cotton bands are properly reproduced by the theory applied for $V_{m n}=0.5$ $\omega$. It is not true for the dashed line spectra calculated for $V_{m n}=0$, i.e. when two peridinin states ${ }^{1} B(m)$ and ${ }^{1} B(n)$ give rise independently to CD spectra of $\mathrm{PCP}$ complexes. This observation allows us to state that the interstate coupling enter as an important ingredient of interactions within excited states manifolds of PCP complex in question. The similar conclusion could be drawn for $\alpha$-crustacyanin pigments containing astaxantin dimers as optically active groups [8].

The large sensitivity of CD spectra to changes of interstate coupling matrix elements $V_{m n}$ may be visualised comparing CD spectra of diffrent PCP complexes. Such a comparision is made in Fig. 4(a) for PCP from A. carlerae and A. rhyncocephalenum dinoflagellate algae. Both PCP $(A . c$.) and PCP $(A . r$.$) complexes$ contain the peridinin dimer but they differ due to number of peridinin molecules per chlorophyll-a. Therefore it would be a reason to suppose that, upon going from PCP $(A . c$.) to PCP $(A . r$.$) , the peridinin dimer accommodated in the$ slighty different environment undergoes small changes of relative orientation of chromophores. If this is the case, then structural changes accompanied by small modifications of interchromophore interactions should account for the rotatory strength redistribution observed in CD spectra shown in Fig. 4(a). Indeed the desired changes are seen in Fig. $4(\mathrm{~b})$ where CD spectra calculated for $V_{m n}=0.5 \omega$ and $\beta=20^{\circ}$ (solid line) and $V_{m n}=0.3 \omega$ and $\beta=30^{\circ}$ (dotted line) are compared. So, it is plausible to consider that the changes in Fig. 4(a) are really due to small structural deformation of peridinin dimer as discussed above.

In our opinion the agreement with experiment as it stands leaves little doubt that the chiral properties of PCP complexes can be reasonably explained by the vibronic dimer theory which includes the.multistate coupling effects in the excited states of peridinin dimer. Some discrepancies between theory and experiment, however, cannot be eliminated in the framework of the theory applied. More specifically the low-energy negative Cotton band in Fig. 3 is structurally too rich compared to the experiment and the same occurs in the high-energy region of the absorption spectra. Since these discrepancies appear in the energy regions which coincide well with $Q_{x}$ and $B_{x}\left(B_{y}\right)$ absorption bands of chlorophyll-a, it is very likely that an inclusion of interaction between peridinin dimer and chlorophyll-a would improve the agreement with experiment. Moreover, such an interaction favored by the coincidence envisaged can be responsible for the efficient energy transfer from 


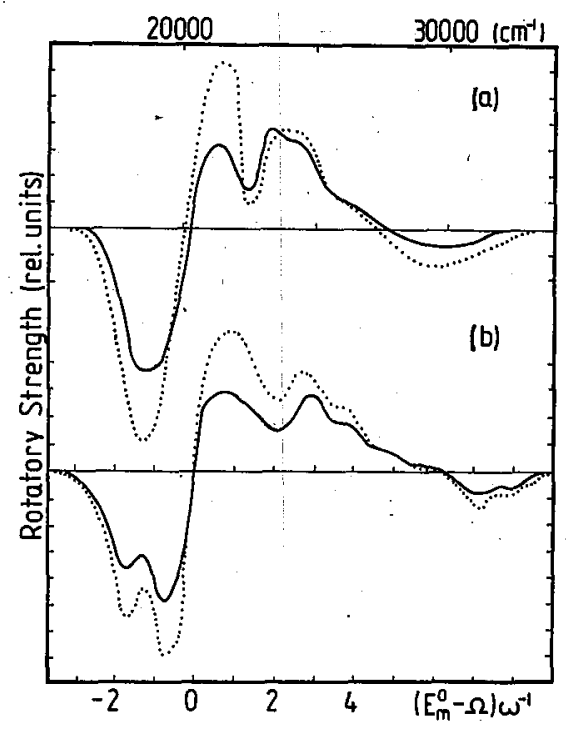

Fig. 4. (a) Experimental CD spectra for A. carterae (-) and A. rhyncocephaleum (...). (b) Theoretical CD spectra calculated for $V_{m n}=0.3 \omega, \beta=30^{\circ}(\ldots)$ and $V_{m n}=0.5 \omega$, $\beta=20^{\circ}(-)$. The other parameters are the same as in Fig. 3.

peridinin dimer to chlorophyll-a occuring in PCP complexes in vivo [5]. In fact, chlorophyll-peridinin dimer interaction can cause that the vibronic levels $\Psi_{\nu, \pm} \mathrm{em}$ bedded in the vibronic manifolds of $Q$ and $B$ states will decay very fast [14] and it will lead to larger values of $\Gamma_{\nu}^{ \pm}$than these used in our calculations. Particulary, it is expected to be true in the energy region corresponding to the $Q_{x}$ state of chlorophyll where the largest discrepancies appear in CD spectra of peridinin dimer. Therefore, we arrive at the conclusion that non-radiative channel(s) $\Psi_{\nu \pm} \rightarrow Q_{x}$ can be very efficient to transfer energy from the peridinin dimer to the chlorophyll in proteins of interest.

Without more detailed and sophisticated theoretical studies nothing more can be said in this matter at present. So, we will not carry this discussion further.

\section{References}

[1] T. Aragò, Mém. Inst. 12, 93 (1811).

[2] J.B. Biot, Mém. Acad. Sci. 2, 41 (1817).

[3] II. Eyring, Ilan-Chung Liu, O. Caldwell, Chem. Rev. 68, 525 (1968).

[4] P. Crabbé, Optical Rotalory Dispersion and Circular Dichroism in Organic Chemistry, IIolden-Day, San Francisco 1965.

[5] Prasad Koka, Pill-Soon Song, Biochim. Biophys. Acla 495, 220 (1977). 
[6] Pill-Soon Song, Prasad Koka, B. Prézelin, F.T. Haxo, Biochemistry 15, $4422(1976)$.

[7] T.Young Lee, J. Jung, Pill-Soon Song, J. Biochem. 88, 663 (1980).

[8] M. Pawlikowski, Acta Phys. Pol. A74, 145 (1988).

[9] S.H. Lin, J. Chem. Phys. 55, 3546 (1971).

[10] R.L. Fulton, M. Gouterman, J. Chem. Phys. 41, 2280 (1964).

[11] S. Czarniecki, A.Witkowski, M. Zgierski, Acla Phys. Pol. A51, 451 (1977).

[12] M.Z. Zgierski, M. Pawlikowski, J. Chem. Phys. 79, 1616 (1983).

[13] M. Pawlikowski, Acta Phys. Pol. A74, 295 (1988).

[14] B.R. Henry, W. Siebrand, Organic Molecular Photophysics, ed.J.B. Birks, Vol. 1, Wiley, New York 1975, p. 153. 